\title{
Sensibilidade de parametrizações de camada limite planetária através do WRF Single-Column Model para o Centro de Lançamento de Alcântara
}

\author{
Sensibility of the planetary boundary layer parameterizations by WRF Single-Column \\ Model in the Alcantara Launch Center
}

\author{
Diogo Nunes da Silva Ramos ${ }^{1}$, Julio Pablo Reyes Fernandez ${ }^{2}$ e Gilberto Fernando Fisch ${ }^{3}$ \\ ${ }^{1}$ Programa de Pós-Graduação em Meteorologia, Instituto Nacional de Pesquisas Espaciais, Cachoeira Paulista - SP, Brasil. \\ ${ }^{2}$ Instituto Nacional de Pesquisas Espaciais, Cachoeira Paulista - SP, Brasil. \\ ${ }^{3}$ Instituto de Aeronáutica e Espaço, Departamento de Ciência e Tecnologia Aeroespacial, São José dos Campos - SP, Brasil.
}

\begin{abstract}
Resumo
Este trabalho avalia a camada limite planetária (CLP) através da sensibilidade de diferentes parametrizações de CLP para o Centro de Lançamento de Alcântara (CLA). O estudo foi produzido usando dois diferentes bancos de dados, um observacional e outro numérico. Os dados observados em superfície e por radiossondas foram da campanha de Alcântara do Projeto CHUVA e aplicados na comparação com as integrações numéricas. Estas integrações foram obtidas com cinco diferentes parametrizações de CLP (YSU, MYJ, QNSE, MYNN2 e TEMF) no modelo WRF (3.7.1) em sua versão de Single-Column Model (SCM), inicializadas com as análises finais do modelo global do NCEP (FNL/GFS) de 18Z do dia 05 de março de 2010, para simulações de 30 horas com spin-up $(6+24$ horas). Os resultados gerais não destacam uma padronização de qualidade de alguma parametrização, apesar que os esquemas MYNN, QNSE e YSU foram mais próximos das observações em diferentes momentos. A determinação da altura da CLP foi melhor simulada pelos esquemas de YSU e QNSE, e MYJ para temperatura do ar em $2 m$. É importante ressaltar as limitações dinâmicas que um modelo SCM possui, adicionando ainda a complexidade do terreno local de Alcântara e suas características costeiras de meso e larga escala (e.g. brisas e ZCIT). Estes problemas motivam a realização de estudos futuros, como a aplicação de modelos tridimensionais de microescala, como o LES.
\end{abstract}

Palavras-chave: Camada limite planetária (CLP). Modelo WRF. Parametrização de CLP.

\begin{abstract}
This study evaluates the planetary boundary layer $(P B L)$ by the sensitivity of different PBL parameterizations for the Alcântara Rocket Launch Center (CLA). The study was produced using two distinct databases, an observational and other numeric. The observational data in surface and radiosondes were of the CHUVA Project for the Alcantara campaign and applied in comparison with the WRF-SCM integrations. These integrations were obtained using five PBL parameterizations (YSU, MYJ, QNSE, MYNN2 and TEMF) in the WRF model (3.7.1) in its version of Single-Column Model (SCM), with initial condition of NCEP's global model final analysis (FNL/GFS) for $18 \mathrm{Z}$ on March 5, 2010, integrating for 30 hours simulations considering spin-up (6 + 24 hours). The overall results do not highlight a standardization of quality of some parameter, despite the MYNN, QNSE and YSU schemes have been closer to the observations at different situations. The PBL height determination was better simulated with YSU and QNSE schemes, and with MYJ to air temperature at $2 m$. Importantly, the dynamic limitations that an SCM model has also adding the complexity of the Alcântara site and its coastal features of meso and large scale (eg breezes and ITCZ). These problems motivates the future studies, for example, with three-dimensional microscale models such as LES.
\end{abstract}

Keywords: Planetary boundary layer (PBL). WRF model. PBL parameterization. 


\section{Introdução}

O aprimoramento dos modelos atmosféricos de previsão numérica de tempo e clima (PNTC) é possível através de estudos com informações observacionais, desenvolvimento de modelos conceituais e experimentos numéricos (Warner, 2011). Nestes modelos, a descrição da turbulência atmosférica é realizada através das parametrizações de camada limite planetária (CLP). Uma parametrização de CLP é responsável por resolver os fluxos turbulentos de calor, umidade e momentum em toda a coluna atmosférica. A composição destes fluxos turbulentos influenciam diretamente na dispersão de poluentes, alterar a espessura da CLP, impacta na formação de nuvens, na segurança de voos, geração de energia eólica, dentre outros. Estas características estão contidas no interior da CLP, assim como a maior parte das atividades humanas. Sendo assim, é importante que estes comportamentos sejam computados de forma adequada nos modelos de PNTC (Baklanov et al., 2011).

A representação destes processos turbulentos em uma alta resolução espaço-temporal eleva o custo computacional dos modelos regionais ou globais de PNTC. Deste modo, a aplicação de modelos em escala micrometeorológica (inferior a $1 \mathrm{~km}$ ) é ainda mais complexa e desafiante. Contudo, uma opção eficaz em modelagem micrometeorológica é o uso de modelos unidimensionais ou de coluna simples (SCM, em inglês). Holtslag e Steeneveld (2011) afirmam que a aplicação de SCM permite identificar as limitações gerais de parametrizações através de testes de sensibilidade. Suas principais vantagens são o baixo custo computacional e simplicidade de configuração para casos idealizados. Contudo, suas principais limitações estão na descrição de processos onde a advecção é importante, além de processos influenciados por mecanismos de meso ou larga escala (e.g. movimentos de subsidência e deslocamento de sistemas precipitantes). Por esta razão, seu uso é recomendado para locais com topografia e vegetação homogênea, além de condições meteorológicas locais próximas da neutralidade. Em cenários onde o terreno não possui estas características, a simulação de grandes vórtices (LES, em inglês) é a alternativa mais adequada (Bosveld et al., 2014), entretanto, sua alta demanda de recurso computacional ainda é um entrave para a maior difusão de estudos deste tipo.

Uma característica comum das parametrizações é seu desenvolvimento através de estudos e medições em latitudes médias em situações atmosféricas e de terreno favoráveis. Com isto, estas parametrizações podem apresentar resultados inadequados para condições atmosféricas adversas e/ou de terreno complexo (Stensrud, 2007). A lacuna do conhecimento na representação numérica da CLP tropical é um fator adicional a este contexto, apesar da sua grande importância nos mecanismos precipitantes (Krishnamurti et al., 2013). A modelagem da CLP tropical é ainda mais desafiante em regiões de terreno complexo, onde o vento próximo da superfície eleva o grau de complexidade dos estudos numéricos (Pires et al., 2015). No Brasil, campanhas experimentais com o estado da arte em instrumentação meteorológica foram realizadas através do Projeto CHUVA, cujo banco de dados vem beneficiando a realização de estudos desta natureza (Machado et al., 2014).

O objetivo deste trabalho é analisar, de forma preliminar, a CLP de Alcântara (MA) através da sensibilidade de diferentes parametrizações turbulentas modelo WRF em sua versão SCM. Este estudo faz uso de dados do Projeto CHUVA para comparação com as simulações do WRF-SCM, em campos de superfície e perfis verticais de temperatura potencial.

\section{Dados e Metodologia}

A área de estudo é o Centro de Lançamento de Alcântara - CLA (MA), local onde lançamentos de foguetes e pesquisas com tecnologia aeroespacial são realizadas. Sua vegetação é tipicamente de restinga, com topografia complexa (presença de falésias com altura média de 40 $\mathrm{m})$ e regime pluviométrico dividido em período seco de julho a dezembro e período chuvoso de janeiro a julho (Fisch, 1999). Dois diferentes conjuntos de dados foram usados neste trabalho, um observacional e outro numérico.

\subsection{Dados observados}

Os dados observados são provenientes da campanha experimental de Alcântara, coletados em março de 2010 durante o Projeto CHUVA (Machado et al., 2014). Os dados selecionados foram de uma estação meteorológica em superfície e de radiossondas (00, 06, 12 e 18Z). O dia selecionado para análise foi 06 de março de 2010, com condições meteorológicas consideradas favoráveis, ou seja, sem precipitação e céu claro. Estas observações serão comparadas com as simulações através dos valores de temperatura em 2 metros $\left(T_{2 m}\right)$ e da altura da CLP $\left(Z_{i}\right)$ obtida pelos perfis verticais de $\theta$. Os valores de $Z_{i}$ foram computados através do método proposto por Liu e Liang (2010)-LL10, que aplica conceitos dos métodos da inversão térmica com o método da parcela de uma forma que permite a automatização de seu cálculo. $\mathrm{O}$ método adota a calibração de sensibilidade dos cálculos através de três parâmetros:

- $\delta_{s}$ - Incremento mínimo de inversão térmica. Este parâmetro é um classificador do tipo de CLP, em função da intensidade da inversão térmica, adotando os seguintes critérios: Se $\Delta \theta\left(=\theta_{5}-\theta_{2}\right)$ for 
$<-\delta_{s}$, assume-se a CLP como convectiva, onde 5 e 2 indicam os níveis verticais $k$, com a superfície em $k=0 ; \Delta \theta>+\delta_{s}=$ CLP estável, $\mathrm{e}-\delta_{S}<\Delta \theta<\delta_{S}$ $=$ CLP neutra. Os valores de $\delta_{s}$ e $\delta_{u}$ propostos por Liu e Liang (2010) foram $1.0 \mathrm{~K}$ e $0.5 \mathrm{~K}$, respectivamente, obtidos através da média de um extenso banco de dados de radiossondagens em diferentes regiões continentais e oceânicas. Neste trabalho, o valor de $\delta_{S}$ foi alterado para $0.5 K$, por ter tido melhor ajuste aos perfis locais de Alcântara.

- $\delta_{u}$ - Incremento mínimo de $\theta$ para a parcela de ar atravessar a inversão abaixo de $Z_{i}$ em situação instável ou neutra. No primeiro caso onde $\theta_{k}-\theta_{1} \geq \delta_{u}$, é então definida esta altura $Z_{k}$ como $Z_{i}$. No caso contrário, aplica-se o algoritmo para CLP estável, onde $Z_{i}$ será obtida através de três possibilidades; 1) $\dot{\theta}_{k}-\dot{\theta}_{k-1}<-\dot{\delta}$; 2) $\dot{\theta}_{k+1}<\dot{\theta}_{r}$; 3) $\dot{\theta}_{k+2}<\dot{\theta}_{r}$. Todas estas condições descrevem o comportamento oscilatório de empuxo. $\dot{\delta}$ representa o parâmetro de curvatura entre os níveis avaliados, definido como constante e igual a $40{\mathrm{~K} . \mathrm{km}^{-1}}^{-}$.

- $\theta_{r}$ - grau de intensidade térmica que a parcela deve ter para atravessar a inversão em $Z_{i}$, ou seja, entrar na zona de entranhamento. Neste trabalhou aplicou-se o mesmo valor proposto pelos autores do método, $4 \mathrm{~K} . \mathrm{km}^{-1}$.

Para atender a sensibilidade do método LL10, a interpolação vertical das radiosondas a cada $5 \mathrm{hPa}$ foi necessário devido à resolução original considerada elevada (inferior a 20m). Segundo os autores do método, este procedimento é requerido para diminuir o impacto das variações verticais, o que elevaria a complexidade na aplicação dos critérios.

Por fim, os valores de Bowen $(\beta)$ analisados, que descreve a razão entre o fluxo de calor sensível $(H)$ e de calor latente $(L E)$, são apenas do WRF-SCM, pois não houve medições destes campos durante o CHUVA. O termo $\beta$ é um indicativo sobre as forçantes do balanço de superfície e, segundo Holtslag e Steeneveld (2011), são índices práticos para análise preliminar de um SCM.

\subsection{Modelo WRF-SCM}

Os experimentos numéricos foram realizados com o modelo WRF (v3.7.1) em sua componente SCM. As condições iniciais do WRF-SCM são as análises finais (FNL) do modelo GFS/NCEP, com resolução espacial de $1^{\circ}$ $x 1^{\circ}$, temporal de 06 horas e com 27 níveis verticais. Foram selecionadas cinco diferentes parametrizações de CLP nas simulações: 1) Yonsei University - YSU; 2) Mellor-Yamada-Janjic - MYJ; 3) Quasi-Normal ScaleElimination - QNSE; 4) Mellor-Yamada Nakanishi-Nino - MYNN2; 5) Total-Energy Mass-Flux (TEMF). As demais parametrizações do WRF-SCM definidas foram as

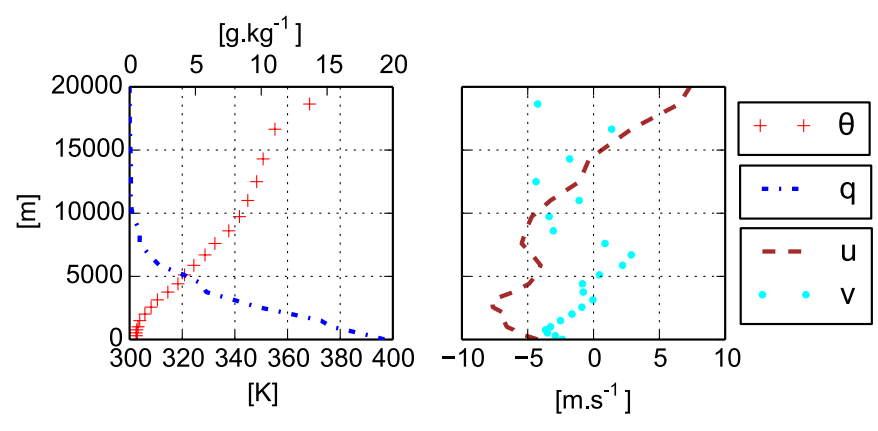

Figura 1: Condições iniciais do WRF-SCM referentes a $18 Z$ de 05/03/2010 obtidas através do préprocessamento das análises finais do modelo global do NCEP.

seguintes: Microfísica - Lin; radiação de onda curta Dudhia; radiação de onda longa - RRTM; superfície NOAH. O esquema de cumulus foi desligado. Com exceção do YSU, os demais esquemas possuem fechamento de turbulência baseado no cálculo da energia cinética turbulenta (com fundamentação no esquema clássico de Mellor-Yamada), com ordem variando entre 1,5 (TEMF e QNSE) a 2,5 (MYJ, TEMF e MYNN2). Dentre os esquemas escolhidos, apenas MYJ e YSU já foram amplamente estudados e aprimorados nos últimos 20 anos, usados desde então para fins operacionais como também de pesquisa. As parametrizações QNSE, MYNN e, principalmente, TEMF são mais recentes, sendo aplicadas apenas para fins de pesquisa. Entretanto, a nova abordagem do QNSE de fechamento de turbulência com formulações independentes do número crítico de Richardson tornam-o um item interessante de estudo, além das suas qualidades já verificadas (Wolff et al., 2011). Existem ainda outras particularidades físicas e numéricas entre os esquemas, cujas referências individuais podem ser obtidas em Skamarock et al. (2015). Como o WRF-SCM não possui sistema de pré-processamento análogo à versão para casos reais, o desenvolvimento de algumas etapas foi necessário para a realização deste estudo. $\mathrm{O}$ WRF-SCM necessita obrigatoriamente de duas condições iniciais, que são as informações de estrutura vertical (altura, componentes do vento, temperatura potencial e umidade específica) e de solo (temperatura da superfície e do solo e da umidade do solo). Os perfis verticais (temperatura, umidade relativa e vento) foram obtidos através da média por área entre pontos de grade próximo ao CLA $\left(2,32^{\circ} \mathrm{S} ; 44,42^{\circ} \mathrm{W}\right)$ a partir das análises finais (FNL) do modelo global (GFS) do NCEP, mostrado na Figura 1. As informações de solo e de larga escala (opcionais no WRF-SCM) foram extraídas através do pré-processamento do WRF por método semelhante aos perfis verticais, mascarando os pontos sobre o oceano. $\mathrm{O}$ movimento vertical, campo considerado na forçante de 

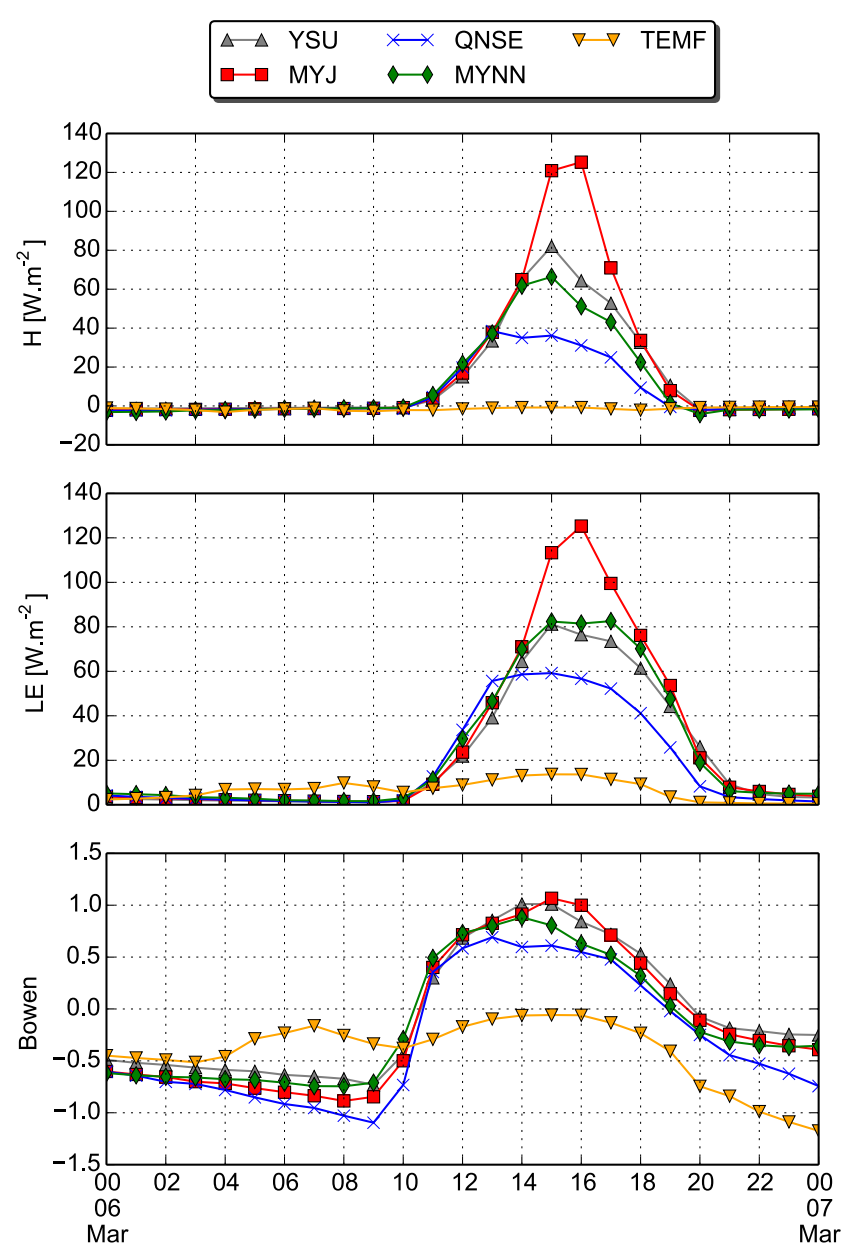

Figura 3: Valores horários de $H, L E$, e $\beta$ computados pelo WRF-SCM.

descrita, inclusive no nível de inversão térmica no topo da CLP noturna, em torno de $100 \mathrm{~m}$. Em aproximadamente $1250 \mathrm{~m}$ de altura, o esquema TEMF não continua seguindo o padrão dos demais esquemas e computa elevados valores de $\theta$. Durante a análise de outros campos simulados com esta opção de CLP (resultados não mostrados), foi observado comportamentos físicos não coerentes com os padrões vistos nos demais esquemas. Uma hipótese é que esta versão do TEMF não tenha sido completamente ajustada para as condições limitantes do SCM do WRF.

O aquecimento superficial e decréscimo da pressão atmosférica neste nível justificam a mudança dos perfis de 12 e $18 \mathrm{Z}$ nos primeiros $500 \mathrm{~m}$. Através deste resultado foi evidente a dificuldade das parametrizações avaliadas em representar a inversão térmica negativa nos 3 primeiros níveis. Esta condição pode explicar a diferença de até $3 K$ entre 0 e $300 m$, uma vez que o cálculo do transporte vertical dos fluxos é diretamente afetado pelos níveis mais baixos da CLP. Mas no modo geral, a tendência de curvatura dos perfis observados foi também repre- sentado pelas simulações, com exceção do TEMF. Tal resultado é importante de ser destacado, uma vez que as condições iniciais do WRF-SCM partiram de análises meteorológicas cujo espaçamento é de aproximadamente $111 \mathrm{~km}$.

No contexto geral, os resultados foram bastante interessantes apesar das várias limitações e considerações empregadas. Por exemplo, foi discutido a formação de CLP interna no CLA devido à presença de falésias e da circulação de brisas, cuja problemática é apresentada por Pires et al. (2015). Estas condições impactam diretamente no escoamento atmosférico sobre o local, alterando os padrões de temperatura através da advecção de umidade do oceano para o continente (Fisch, 1999). Algumas destas limitações encontradas nas parametrizações (transição entre noite e dia, inversão térmica próxima da superfície, dentre outras) são conhecidas e vêm sendo investigadas pela comunidade científica (Bosveld et al., 2014; Holtslag et al., 2013). Stoll e PortéAgel (2009) confirmaram através de LES que pequenas alterações no gradiente vertical de temperatura e nos fluxos superficiais podem impactar significativamente na estrutura vertical da CLP. A geografia complexa e formação de CLP interna do CLA implicam na dificuldade de comparação das simulações com as observações, criando então a necessidade de estudos futuros com outros tipos de modelos. A aplicação do LES com topografia e vegetação em altíssima resolução pode ser promissora na investigação da CLP de CLA. Além disto, durante a campanha experimental de Alcântara do Projeto CHUVA houve a passagem de sistemas meteorológicos adversos, o que pode justificar a realização de estudos de caso para outros dias desta campanha.

\section{Conclusões}

A realização deste trabalho permitiu identificar, de forma preliminar, as principais características e limitações de cinco parametrizações de CLP no modelo WRF-SCM para o Centro de Lançamento de Alcântara. Apesar dos aspectos geográficos complexos do CLA, as simulações tiveram resultados gerais satisfatórios, com características positivas e limitações pontuais em períodos do dia investigado. Os valores simulados de $T_{2 m}$ foram mais próximos das medições durante o dia, com leve vantagem do esquema MYJ. A estrutura vertical simulada da CLP apresentou padrões distintos entre dia e noite, onde os melhores resultados foram para QNSE e YSU, principalmente abaixo de $1000 \mathrm{~m}$. A determinação de $Z_{i}$ pelo TEMF foi ineficaz durante a noite em função da ausência de um tratamento específico CLP estável, o que impactou diretamente ao longo das integrações seguintes. Para os padrões em superfície, os esquemas MYJ e MYNN indicaram maior disponibilidade de energia 
larga escala além do vento geostrófico, foi definido como nulo neste trabalho. As configurações do tipo de vegetação e de solo foram ajustadas seguindo as informações obtidas pelo pré-processamento do WRF com dados do MODIS em 30 arcsegundos de resolução espacial.

Além das configurações de pré-processamento do WRF-SCM, algumas outras considerações foram assumidas. O tempo de amortização dos ruídos das condições iniciais (spin up time) foi de 6 horas para um tempo total de 30 horas de integração. A resolução temporal das integrações foi de 20 segundos, com domínio horizontal de $4 \mathrm{~km} \times 4 \mathrm{~km}, 60$ níveis verticais e topo do modelo em $12 \mathrm{~km}$ de altura.

\section{Resultados e discussões}

A Figura 2 faz referência às simulações de $T_{2 m}$ e a altura da CLP $\left(Z_{i}\right)$. Na comparação com as observações, verifica-se que os valores de $T_{2 m}$ possuem três características distintas e coincidentes com as fases de evolução diurna da CLP. No período noturno, a observação (OBS) de $T_{2 m}$ indica um comportamento estável, com leve resfriamento entre 07 e $10 \mathrm{Z}$ (04 a 07 hora local - HL), sendo um período de transição entre CLP estável e CLP convectiva. Neste intervalo de tempo, todos as simulações mostraram uma tendência de resfriamento contínuo, desde o ocaso (21 Z) ao amanhecer (09 Z). Durante o dia, principalmente entre 14 e $18 \mathrm{Z}$, esta diferença de temperatura entre OBS e WRF-SCM foi menor para o esquema MYJ, seguido do YSU e MYNN. As primeiras 10 horas do dia selecionado para análise mostraram uma diferença baixa entre os cinco esquemas avaliados, divergindo apenas com inicio do dia, principalmente o esquema TEMF e QNSE. É provável que as soluções numéricas adotadas por estes dois esquemas para o aquecimento da superfície e a sua resposta nos fluxos verticais turbulentos tenha impactado neste resultado.

Os resultados da Figura 3 mostram a evolução horária de $H, L E$ e $\beta$. A variação destes fluxos no período do dia (entre 9 e $20 \mathrm{Z}$ ) indicam que a maior parte da energia local é transferida para a atmosfera na forma de calor latente (evaporação do ar). Este comportamento é natural de regiões costeiras tropicais (Stull, 1988), sendo então condizentes com o cenário de CLA. Este resultado é importante pois, segundo Holtslag e Steeneveld (2011), é fundamental que a partição de energia próximo da superfície seja computada de forma adequada para que o desenvolvimento da CLP seja descrito com maior acurácia.

Os valores de $Z_{i}$ mais próximos das observações foram aqueles simulados pela parametrização QNSE, seguida por YSU (menor erro em $12 \mathrm{Z}$ ). Para as $18 \mathrm{Z}$, estes esquemas apresentaram diferenças inferiores a $50 \mathrm{~m}$, abaixo da margem de erro do método LL10 (Liu e Li-
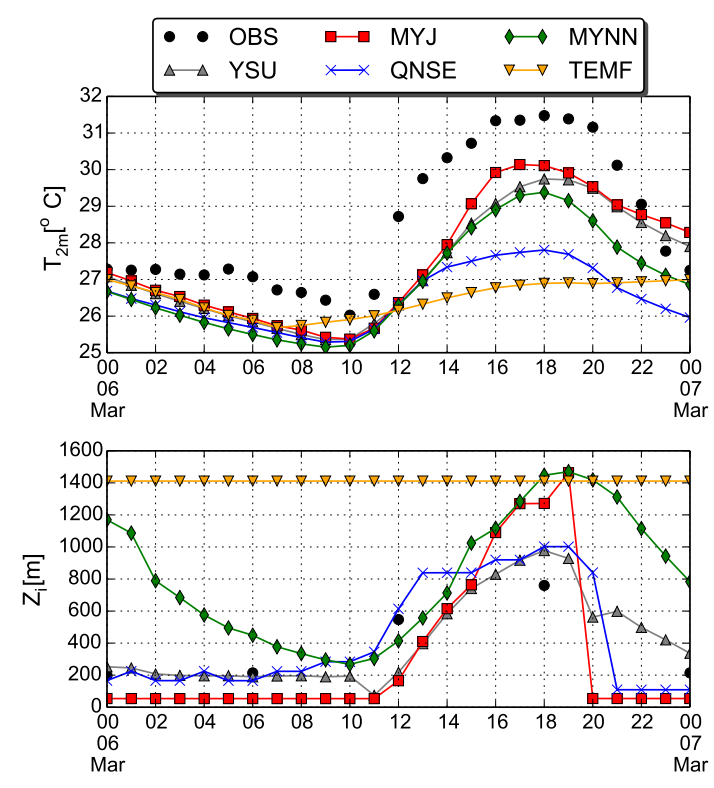

Figura 2: Valores horários da $T_{2 m}$ e $Z_{i}$ obtidas através das simulações do WRF-SCM para 06 de março de 2010.

ang, 2010). Vale ressaltar a evolução de $Z_{i}$ representada por estas duas parametrizações é bastante próxima das reportadas para CLA, cuja altura quase não ultrapassa $1 \mathrm{~km}$ em função das brisas e sua consequente CLP interna (Pires et al., 2015). Os esquemas TEMF e QNSE usam a técnica de difusividade vertical por fluxo de massa (EDMF, em inglês) e suas formulações. O EDMF considera um termo adicional de transporte vertical de massa (seca ou úmida) ao longo da estrutura vertical da CLP. Esta adoção é apropriada para descrição de nuvens estratiformes e de CLP estável. Entretanto, a notável discrepância de $Z_{i}$ durante a noite descrita pelo TEMF é uma das suas limitações mais acentuadas (Angevine et al., 2010). Já o QNSE possui tratamento específico para CLP estável através do filtro temporal de ruídos turbulentos gerados pela estratificação térmica, o que pode justificar a sua qualidade ao apresentar um viés inferior a $50 \mathrm{~m}$ durante a noite. Contudo, deve ser considerado que estes resultados são correspondentes a um único dia de simulação, com uma série de considerações adotadas e limitações conhecidas. Logo, estudos futuros mais amplos são necessários, pois a campanha de Alcântara possuiu pouco mais de 20 dias ininterruptos de radiossondas com diferentes padrões atmosféricos, podendo mostrar outros comportamentos físicos não observados neste trabalho.

A Figura 4 apresenta os perfis verticais de $\theta$ com os diferentes esquemas de CLP investigados no WRFSCM. É perceptível a concordância entre os esquemas para os primeiros $1000 \mathrm{~m}$ em 00 e $06 \mathrm{Z}$, com exceção do TEMF neste último horário. Nestes horários, os perfis foram concordantes com as observações até a altura 

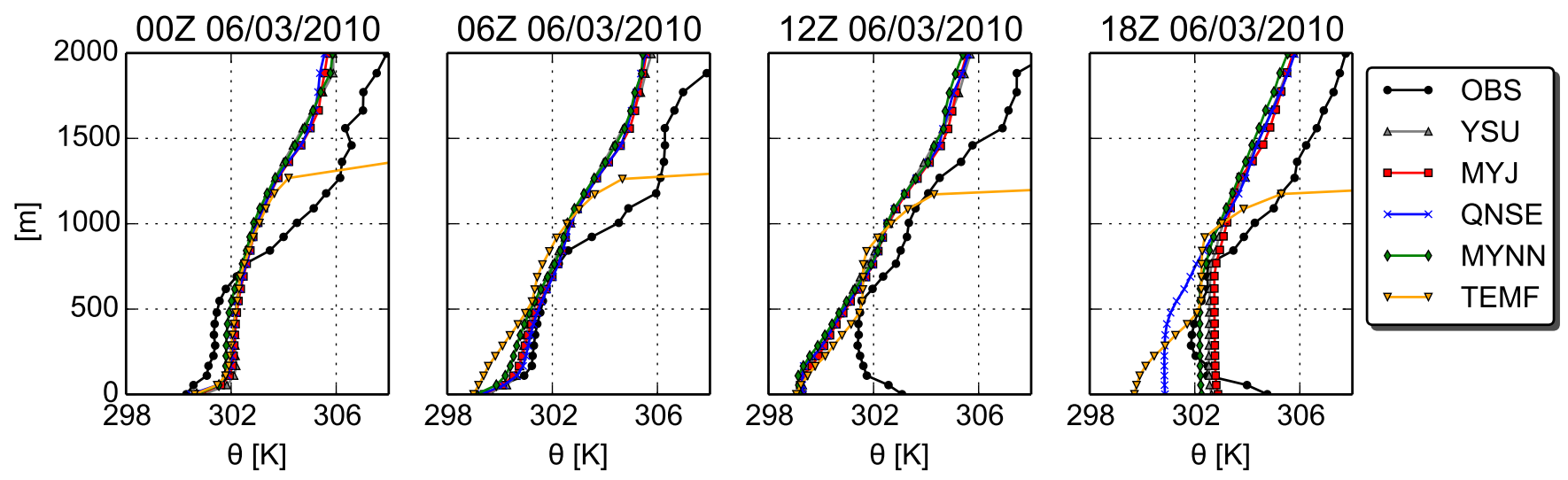

Figura 4: Perfis verticais observados e simulados de $\theta$ para 00, 06, 12 e 18 do dia 06/março/2010.

para aquecimento e evaporação do ar, o que pode ter influenciado no maior erro de $Z_{i}$. A eliminação de ruídos no transporte turbulento descrito pela parametrização QNSE durante a integração é uma característica que também pode justificar sua qualidade nos resultados. Por fim, as limitações de um modelo SCM, a complexidade do terreno e a formação de CLP interna sugerem a necessidade de um estudo mais profundo sobre o escoamento atmosférico no CLA com aplicação de outras ferramentas numéricas, como o LES.

\section{Agradecimentos}

Os autores agradecem ao Projeto CHUVA (FAPESP 15235-8/2009) pela disponibilidade dos dados usados neste trabalho. $\mathrm{O}$ primeiro autor agradece a CAPES e ao CNPq pelo auxílio financeiro concedido através da bolsa de doutorado (número 140797/2015-7) e também ao Dr. Joshua Hacker (National Center for Atmospheric Research NCAR, EUA) pelo auxílio dado no uso do WRF-SCM. $\mathrm{O}$ terceiro autor também agradece ao $\mathrm{CNPq}$, pela bolsa PQ (número 308011/2014-7).

\section{Referências}

Angevine, W. M., Jiang, H., Mauritsen, T. (2010). Performance of an Eddy Diffusivity-Mass Flux Scheme for Shallow Cumulus Boundary Layers. Monthly Weather Review, 138(7), 2895-2912.

Baklanov, A. a., Grisogono, B., Bornstein, R., Mahrt, L., Zilitinkevich, S. S., Taylor, P., Larsen, S. r. E., Rotach, M. W., Fernando, H. J. S. (2011). The Nature, Theory, and Modeling of Atmospheric Planetary Boundary Layers. Bulletin of the American Meteorological Society, 92(2), 123-128.

Bosveld, F. C., Baas, P., Steeneveld, G. J., Holtslag,
A. A. M., Angevine, W. M., Bazile, E., de Bruijn, E. I. F., Deacu, D., Edwards, J. M., Ek, M., Larson, V. E., Pleim, J. E., Raschendorfer, M., Svensson, G. (2014). The Third GABLS Intercomparison Case for Evaluation Studies of Boundary-Layer Models. Part B: Results and Process Understanding. Boundary-Layer Meteorology.

Fisch, G. (1999). Características do perfil vertical do vento no Centro de Lançamento de Foguetes de Alcântara (CLA). Revista Brasileira de Meteorologia, 14(1), 11-21.

Holtslag, A. A. M., Steeneveld, G. J. (2011). Single Column Modeling of Atmospheric Boundary Layers and the Complex Interactions with the Land Surface. Em: Meyers, R. A. (ed) Extreme Environmental Events, Springer New York, New York, NY, pp. 844-857.

Holtslag, A. A. M., Svensson, G., Baas, P., Basu, S., Beare, B., Beljaars, A. C. M., Bosveld, F. C., Cuxart, J., Lindvall, J., Steeneveld, G. J., Tjernstrom, M., Van de Wiel, B. J. H. (2013). Stable Atmospheric Boundary Layers and Diurnal Cycles - Challenges for Weather and Climate Models. Bulletin of the American Meteorological Society, 94, 1691-1706.

Krishnamurti, T. N., Stefanova, L., Misra, V. (2013). Tropical Meteorology: An Introduction. Springer.

Liu, S., Liang, X. Z. (2010). Observed Diurnal Cycle Climatology of Planetary Boundary Layer Height. Journal of Climate, 23(21), 5790-5809.

Machado, L. A. T., Dias, M. A. F. S., Morales, C., Fisch, G. (2014). The Chuva Project - How does convection vary across Brazil? Bulletin of the American Meteorological Society, 95(9), 1365-1380.

Pires, L. B. M., Fisch, G., Gielow, R., Souza, L. F., Avelar, A. C., Paula, I. B. D., Girardi, R. D. M. (2015). A Study 
of the Internal Boundary Layer Generated at the Alcantara Space Center. American Journal of Environmental Engineering, 5(1A), 52-64.

Skamarock, W. C., Wang, W., Bruyère, C., Duda, M., Dudhia, J., Gill, D., Kavulich, M., Keene, K., Lin, H. C., Michalakes, J., Rizvi, S., Zhang, X., Berner, J., Fossell, K. (2015). Weather Research \& Forecasting - ARW Version 3 Modeling System User's Guide. Relatório Técnico, National Center for Atmospheric Research, Boulder, Colorado (EUA).

Stensrud, D. J. (2007). Parameterization Schemes: Keys to Understanding Numerical Weather Prediction Models. Cambridge University Press.

Stoll, R., Porté-Agel, F. (2009). Surface Heterogeneity Effects on Regional-Scale Fluxes in Stable Boundary Layers: Surface Temperature Transitions. Journal of the Atmospheric Sciences, 66(2), 412-431.

Stull, R. B. (1988). An Introduction to Boundary Layer Meteorology. Springer.

Warner, T. T. (2011). Numerical Weather and Climate Prediction. Cambridge University Press.

Wolff, J., Nance, L., Gotway, J. H., Oldenburg, P., Harrold, M., Trabold, Z. (2011). WRF QNSE test and evaluation. URL http://www. dtcenter.org/eval/meso_ $\bmod /$ afwa_test/wrf_v3.2.1/index.php. 